\title{
CHILDHOOD DEATHS FROM MALIGNANT NEOPLASMS IN ACCRA
}

\author{
"R.K. GYASI and Y. TETTEY \\ Departments of Pathology, University of Ghana Medical School, P. O. Box 4236, Accra, Ghana
}

\section{SUMMARY}

Background: Malignant neoplasms are set to become a leading cause of childhood death in subSaharan Africa as immunization programmes reduce deaths due to infectious diseases. Knowledge of the pattern of deaths from these neoplasms is therefore desirable.

Objective: To describe the pattern of deaths from paediatric malignancies, compare this to morbidity figures and provide baseline data for planning child care services.

Methods: A 10 year retrospective survey of autopsy cases of paediatric malignancies at the Korle-Bu Teaching Hospital Mortuary was carried out based on autopsy files form January 1, 1990 to December 31, 1999

Results: A total of 252 cases of childhood malignant tumours were retrieved, 139 males and 113 females with a male to female ratio of 1.2:1. The most common malignancy was lymphoma forming $54 \%$ of cases and almost all of Non-Hodgkin's type. Non-Burkitts type (29\%) was the commonest followed by Burkitts (24\%) and Hodgkin's disease only one percent. The lymphomas were followed by central nervous system (CNS) tumours $(13 \%)$, nephroblastomas $(10.3 \%)$ leukemias $(6.7 \%)$ hepatic tumours $(4 \%)$ and sarcomas $(2.6 \%)$. Less common tumours were Neuroblastomas $(2.4 \%)$ and retinoblastomas (2\%).

Conclusion: Overall the pattern of deaths from paediatric malignancies followed the pattern of relative incidence in morbidity figures from Ghana and the subregion except for a relatively higher proportion of deaths from CNS tumours and a lower proportion from sarcomas. The pattern of cancer deaths seen in this study is similar to that seen in advanced countries except that lymphomas replace leukemia as the commonest cause of death

Keywords: Autopsy, childhood deaths, malignant neoplasms.

\section{INTRODUCTION}

Malignancies have been considered an uncommon cause of death in sub-Saharan Africa forming $1.25 \%$ of clinical cause of childhood death and $0.5 \%$ of autopsy cases seen at the Korle-Bu Hospi$\mathrm{tal}^{1,2}$. With the increasing success of the Expanded Programme on Immunisation (EPI) and some improvement in socio-economic status, there has been a reduction of childhood infectious diseases, therefore, paediatric non-communicable diseases including malignancies have become increasingly important.

A survey of childhood malignancies at the KorleBu Hospital by Welbeck and Hesse ${ }^{3}$ showed increasing incidence of paediatric malignancies. It is expected that as countries in the sub region approach middle income status, malignancies will become a leading cause of death as has occurred in some countries ${ }^{4}$.

This survey of autopsy cases aims to highlight the pattern of deaths due to paediatric malignancies and to show any differences that exist when compared to reported morbidity figures so that different approaches could be taken to improve detection and diagnosis of paediatric malignancies.

\section{PATIENTS AND METHODS}

The Korle-Bu Teaching Hospital is the main referral center for the southern part of Ghana. Autopsy services at the Korle-Bu Hospital include hospital autopsies and medico-legal cases from the Accra Metropolis, and southern Ghana. Autopsy files of all malignancies were retrieved for the period January 1, 1990 to December 31, 1999. Data on age, gender, diagnosis including histologic type, site and spread of malignancy in children aged less than 15 years were collected and entered into a database and analysised using EPI-INFO 2000 soft ware ${ }^{5}$.

\section{RESULTS}

* Author for correspondence

rkg539us@yahoo.com 
A total of 252 cases of malignancies in the paediatric group occurred out of a total of 17,500 autopsies and 1,535 malignancies over the period constituting a relative prevalence of $1.4 \%$ of autopsies and $16.4 \%$ of autopsy malignancies. Most of the cases (186/252), 74\% were medico-legal and $26 \%$ hospital autopsies. There were 139 males $(55 \%)$ and 113 females (45\%) giving an $\mathrm{M}$ : F ratio of 1.23:1. Most children with malignancies were aged 5-9 years (37\%) Table1. The least represented were the $0-4$ year group (24\%). The modal age of death from childhood cancer was 6 years for males and 11 years for females.

Table 1 Frequency of paediatric malignancies: 1990 to 1999.

\begin{tabular}{lcc}
\hline Histologic Type & Number & Frequency \% \\
\hline Lymphomas & 135 & 53.6 \\
CNS tumours & 32 & 12.7 \\
Nephroblastoma & 26 & 10.3 \\
Leukemias & 17 & 6.7 \\
Hepatic tumours & 10 & 4 \\
Sarcomas & 8 & 3.2 \\
Neuroblastoma & 6 & 2.4 \\
Retinoblastoma & 5 & 2 \\
Others & 13 & 5.1 \\
\hline Total & $\mathbf{2 5 2}$ & $\mathbf{1 0 0}$
\end{tabular}

Table 2 Age/gender distribution of autopsied padiatric malignancies

\begin{tabular}{lccccc}
\hline \multicolumn{1}{c}{ Type } & $\begin{array}{c}\text { M/F } \\
\text { ratio }\end{array}$ & $\mathbf{0 - 4}$ & $\begin{array}{c}\mathbf{5}- \\
\mathbf{9}\end{array}$ & $\begin{array}{c}\mathbf{1 0}- \\
\mathbf{1 4}\end{array}$ & Total \\
\hline $\begin{array}{l}\text { Non-Hodgkin's } \\
\text { Lymphoma }\end{array}$ & $1.25: 1$ & 13 & 29 & 30 & 72 \\
$\begin{array}{l}\text { Burkitt's Lym- } \\
\text { phoma }\end{array}$ & $1.1: 1$ & 12 & 33 & 16 & 61 \\
$\begin{array}{l}\text { Hodgkin's Dis- } \\
\text { ease }\end{array}$ & $2: 0$ & - & - & 2 & 2 \\
$\begin{array}{l}\text { Intracranial } \\
\text { Tumours }\end{array}$ & $1.13: 1$ & 10 & 9 & 12 & $31+1^{*}$ \\
Nephroblastoma & $1.36: 1$ & 16 & 7 & 3 & 26 \\
Leukemias & $1: 1$ & 3 & 9 & 5 & 17 \\
Hepatic tumours & $2: 1$ & 6 & - & 4 & 10 \\
Sarcomas & $1.6: 1$ & 5 & 1 & 2 & 8 \\
Neuroblastoma & $2: 1$ & 2 & 3 & 1 & 6 \\
Retinoblastoma & $4: 1$ & 4 & 1 & - & 5 \\
Others & $1.6: 1$ & 4 & 6 & 3 & 13 \\
\hline Total & $\mathbf{1 . 2 3 : 1}$ & $\mathbf{7 5}$ & $\mathbf{9 8}$ & $\mathbf{7 8}$ & $\mathbf{2 5 2}$
\end{tabular}

Table 2 shows the male:female ratios and age distributions of the different types of malignancies.
Lymphomas: Non-Hodgkin's Non-Burkitt's type lymphomas (72) formed the most common lymphoma $(53.3 \%)$ and the single most common paediatric malignancy (29.6\%). Burkitt's Lymphomas formed $45.2 \%$ of lymphomas and $24 \%$ of paediatric malignancies. The rest of the lymphomas were Hodgkin's type (1.5\%). The modal age group of death for the non-Hodgkin's, non-Burkitt's lymphomas was 10-14 with modal age of 11 years. For Burkitt's lymphoma, the modal age group was 5-9 years with the modal age of six. Overall, there was a slight male preponderance over females of $1.25: 1$

Central Nervous System (CNS) Tumours were the second commonest cause of death from malignancy in children (13\% of cases). The modal age group was 11-14 years with modal age of 11 years.

The CNS sites were cerebral, cerebellar, brainstem and pituitary. Histologic types were gliomas (21), meningiomas (2), and pituitary adenoma (1). The histologic type was not stated in $25 \%(8 / 32)$ of cases. The Male:Female ratio for CNS tumours was $1.14: 1$.

Nephroblastomas (26) were the third commonest tumours forming $10 \%$ of cases. The modal age group was $0-4$ years with the modal age of occurrence of 4 years. The Male:Female ratio was 1.2:1.

The leukaemias (17) were the fourth commonest (6.7\%). Modal age group 5-9 years and peak age was 7years and the m:f ratio 1:1. Acute lymphoblastic leukaemia was more common (80\%) than acute myeloid leukemias. There was no chronic leukaemia among the autopsied cases.

Tumours of the liver (10) were the fifth commonest tumours $(4 \%)$. There were two distinct age groups according to types of tumour. Hepatoblastomas occurred among 0-4 year group with peak age of less than 1 year. Hepatocellular carcinomas occurred among 10-14 year group. Overall the male:female ratio for hepatic tumours was $2: 1$.

Eight cases of sarcomas were recorded (3.2\%) making sarcomas the sixth commonest tumour. They occurred evenly over the age groups with a peak of 4 years and m:f ratio of 1.6:1. The histologic types were rhabdomyosarcoma(3), osteosarcoma(2) and Ewingssarcoma(1).

Neuroblastomas (6) were the seventh commonest tumour and formed $2.4 \%$ of tumours and occurred 
mainly in the first and second quinqueniums with a peak age of 8 years.

Retinoblastomas (5) were eighth (2\%). Almost all occurred in the 0-4 year group. Males were more common with m:f ratio $4: 1$.

Other tumours (13) formed 5.2\% of total tumours. These included nasopharyngeal carcinomas (3), cardiac tumours (2), ovarian teratocarcinomas (2), retroperitoneal tumours (3), soft tissue tumours of abdomen (1), carcinomatosis (1) and thymic tumours (2).

The five commonest tumours in descending order of frequency for the 0-4 year group were lymphomas, nephroblastoma, CNS tumours, hepatic tumours and sarcomas. For the 5-9 year group: lymphomas, CNS tumours, leukaemia, nephroblastomas and neuroblastoma. The 10-14 year group had lymphoma, CNS tumours, leukemias, hepatic tumours and nephroblastomas (Table 2).

\section{DISCUSSION}

Over the 10-year period under review, 252 pediatric malignancies were seen at the Korle Bu Hospital mortuary. The average of 25 autopsied cases of malignant tumours per year is about a third of the expected number of cases at the Korle-Bu Hospital in a year ${ }^{3}$, and reflects a low autopsy rate in the hospital. However since many of the cases $(78 \%)$ were medico-legal cases directly from the community it may reflect also a high proportion of undiagnosed malignancy among the general populace only picked up at autopsy.

The male preponderance is consistent with surveys on cancer incidence in Ghana, the sub-region and elsewhere $^{1-4,6-8}$. The relative frequency of paediatric malignancies of $1.4 \%$ of autopsies was almost three times that reported about forty years ago $(0.53 \%)^{2}$ and indicates the increasing trend of malignancies as a cause of death in children.

The commonest childhood malignancy in this study was malignant lymphoma making up 54\% of the total. This is in keeping with morbidity surveys in Ghana and in the subregion ${ }^{3-7,9}$ and different from advanced countries like the US where leukaemia is the commonest malignancy ${ }^{8}$ and lymphomas are the third commonest cause of death ${ }^{9}$. An additional reason is the presence of a Burkitt's tumour project at Korle Bu. The apparent higher proportions of non-Burkitt's type Non-Hodgkin's lymphomas, was surprising in view of the presence of the Burkitt's tumour project in the Hospital.
This may be a true reflection of the proportions of deaths due these tumours, or to better treatment outcomes among Burkitt's lymphoma cases compared to the other non-Hodgkin's lymphomas or it may be because fewer cases of diagnosed Burkitt's lymphoma were sent for autopsy. A proper register of tumours and subsequent epidemiological studies will be required to answer such questions.

CNS tumours were the second commonest lesion at autopsy (13\%). This is different from morbidity figures from Ghana and the subregion ${ }^{3,7}$ with an incidence of $1.2 \%$ and $0.6 \%$ respectively and rather similar to the $\mathrm{US}^{8}$. The difference may reflect either the difficulties encountered in the diagnosis of CNS tumours so that many are only picked up at autopsy or the increased capture of deaths of cases referred to the few neurosurgeons available in Korle $\mathrm{Bu}$ and elsewhere in Accra. Most of the cases occurred among children who died on wards at the Korle $\mathrm{Bu}$ Teaching Hospital and seems to support the latter explanation.

Nephroblastomas were the third commonest tumours as expected from the morbidity figures of between $8-14 \%$ in Ghana, the sub-region and elsewhere ${ }^{3,8,9}$.

Leukemias were the fourth commonest tumours and occurred most commonly in the 5-9 year agegroup as expected from the relative position in earlier morbidity figures from Accra and elsewhere in West Africa ${ }^{3,6,9}$. This pattern is different from developed countries where Leukemia is a more common cause of death ${ }^{7}$.

Hepatic tumours were the fifth commonest tumours as expected from the relative position earlier morbidity figures from Accra and elsewhere in West Africa ${ }^{3,5,7,9}$.

Sarcomas were the sixth commonest malignancy and a less common cause of death $(3.2 \%)$ as expected from the incidence of $3 \%$ in Ghana 3 though it is a more common malignancy in the subregion ${ }^{7}$.

Neuroblastoma were the seventh commonest malignancy and a less common cause of death as expected from the incidence of $1-2 \%$ in the subregion $^{3,6,9}$.

Retinoblastomas were the eighth commonest malignancy causing death though the second commonest tumour in patients presenting at Korle-Bu. This finding may be as a result of successful 
treatment or that autopsy is not requested for most of the deaths in diagnosed cases.

The five most common tumours varied slightly for the three quinqueniums. The first quinquenium had lymphomas as the commonest tumour but as expected embryonal tumours were the next commonest types of tumours; from the kidneys (nephroblastomas) and the liver. The sarcomas that followed were also mostly embryonal type tumours as expected. For the second and third quinqueniums, embryonal tumours from the kidney and liver became less common as compared to CNS tumours and leukemias. Also apart from the reversal of positions of lymphomas and leukemias in these two quinqueniums, the relative frequencies of the top three tumours were the same as that for the advanced countries like the $\mathrm{US}^{10}$.

\section{CONCLUSION}

Malignant lymphomas are the commonest cause of cancer death in children under 15 years of age. This finding is different from that of advanced countries where leukemias are the most common cause of death.

Overall, the major types of tumours causing death followed the pattern of relative incidence in morbidity figures from Ghana and the sub-region except for a relatively higher proportion of deaths from CNS tumours and a lower proportion from sarcomas. The pattern seen in this study is similar to causes of cancer deaths in advanced countries except that lymphomas replace leukaemias as the commonest cause of death.

Further epidemiologic studies are needed to establish and explain the apparent differences between morbidity and mortality patterns of childhood malignancies and such studies would require the support of an effective Cancer Registry.

\section{REFERENCES}

1. Commey JOO. Childhood mortality at the Korle Bu Hospital, Accra, 1986-1987. Ghana Med J 1991; 25: 334-341.

2. Christian EC. Why do our children die? Ghana Med J 1967; 7: 111-114.

3. Welbeck JE, and Hesse AA. Pattern of childhood malignancy in Korle Bu Teaching Hospital, Ghana. West Afr Med J 1998; 17(2):8184.

4. Wagner HP, and Antic V. The problem of paediatric malignancies in the developing world. Ann N Y Acad Sci 1997; 824: 193-204.

5. U.S. Dept. of Health and Human Services, Public Health Service, Centers for Disease Control. EPI INFO [Computer file]. Atlanta, GA 1990.

6. Akang EEU. Tumors of childhood in Ibadan, Nigeria 1973-1990. Paed Path and Lab Medi 1996; 6: 791-800.

7. Augua NEN, and Okeahialam T. Malignant diseases of childhood seen at the University of Nigeria Teaching Hospital (UNTH), Enugu, Nigeria.

8. Young JL, Muller RW. Incidence of malignant tumors in US children. $J$ Paediatrics $1975 ; 2: 245-258$.

9. Onwasigwe CN, Aniebue PN, Ndu AC. Spectrum of paediatric malignancies in Eastern Nigeria 1989-1998. West Afr Med J 2002; 21(1): 31-33.

10. Surveillance Epidemiology and End Results from 1975-2000 Division of Cancer control and Population Sciences. National Cancer Institute Reports 2003 presentation by American Cancer Society 2004; 23. 